DIW BERLIN

Discussion

Papers

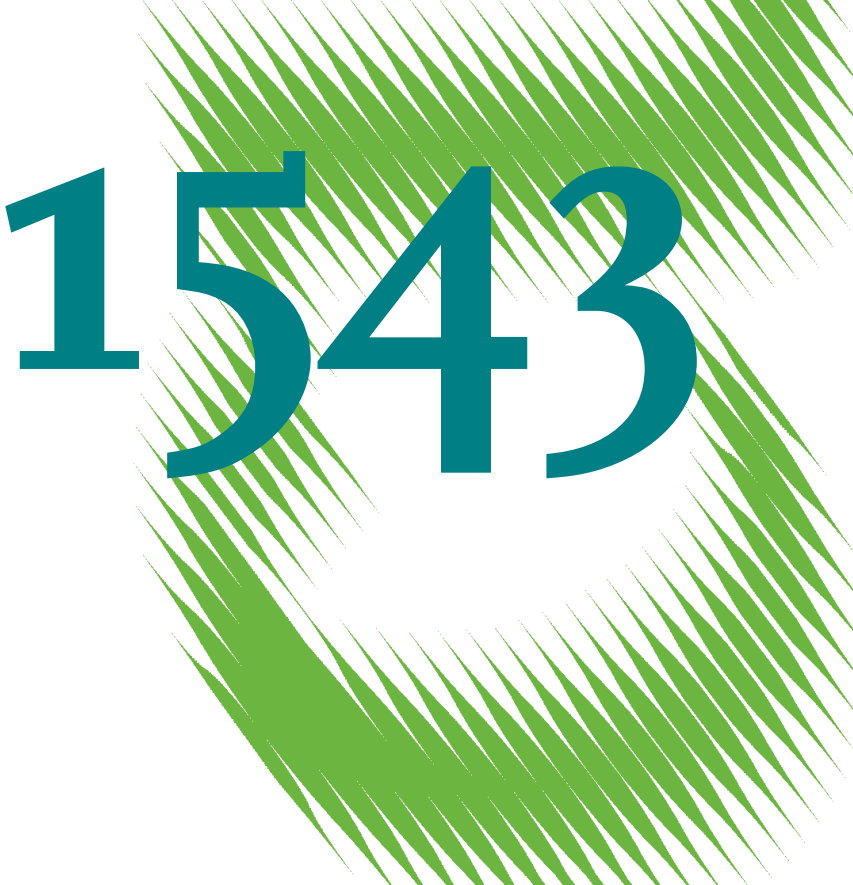

Information Acquisition in Vertical Relations 
Opinions expressed in this paper are those of the author(s) and do not necessarily reflect views of the institute.

IMPRESSUM

(C) DIW Berlin, 2016

DIW Berlin

German Institute for Economic Research

Mohrenstr. 58

10117 Berlin

Tel. +49 (30) $89789-0$

Fax +49 (30) $89789-200$

http://www.diw.de

ISSN electronic edition 1619-4535

Papers can be downloaded free of charge from the DIW Berlin website:

http://www.diw.de/discussionpapers

Discussion Papers of DIW Berlin are indexed in RePEc and SSRN:

http://ideas.repec.org/s/diw/diwwpp.html

http://www.ssrn.com/link/DIW-Berlin-German-Inst-Econ-Res.html 


\title{
Information Acquisition in Vertical Relations
}

\author{
Pio Baake \\ DIW Berlin*
}

\author{
Andreas Harasser \\ DIW Berlin ${ }^{\dagger}$
}

\author{
Friederike Heiny \\ Humboldt University Berlin $\ddagger$
}

January 28, 2016

\begin{abstract}
We analyze a simple supply chain with one supplier, one retailer and uncertainty about market demand. Focusing on the incentives of the supplier and the retailer to enhance their private information about the actual market conditions, we show that choices on information acquisition are strategic complements. While the retailer's incentives are mainly driven by the information rent that he can earn, the supplier will choose to acquire information only if the retailer is rather well informed, even though the information is free of charge.
\end{abstract}

JEL-classification numbers: D82, D83, D86

Keywords: asymmetric information, information acquisition, vertical relations

*DIW Berlin, Mohrenstr. 58, 10117 Berlin, Germany, Email: pbaake@diw.de.

${ }^{\dagger}$ DIW Berlin, Mohrenstr. 58, 10117 Berlin, Germany, Email: aharasser@diw.de.

${ }^{\ddagger}$ Faculty of Economics and Business Administration, Email: friederike.heiny@student.hu-berlin.de 


\section{Introduction}

Uncertainty about demand is a general phenomenon in markets for new products or in markets where consumer preferences tend to vary with time, e.g., markets for fashion goods. Similarly, exogenous demand shocks can lead to price fluctuations and thus expose firms to high economic risks. Information obtained from market research can reduce the firms' uncertainty. In supply chains the question arises whether suppliers and/or retailers want to acquire information about market demand. There are two main strategic issues involved: First, suppliers can use the contracts they offer to signal their private information. Second, retailers can increase their information rent, if they reduce their uncertainty.

We analyze a simple model with one supplier, one retailer and uncertainty about demand in a perfectly competitive market. The uncertainty is modelled as an uncertainty about the price, at which the retailer can sell the supplier's product and which might be either high or low. The supplier and the retailer receive private signals about the actual price of the product. The precision of these signals, i.e., the probability with which they signal the actual price, can be chosen by each of the players. Although the signals themselves are private, we assume that the choices on signal precision are observable. The supplier offers contracts to the retailer, which each specify a certain quantity and transfer. The contracts may depend on the supplier's private information, which has a signalling effect towards the retailer. The retailer chooses one of the offered contracts, given his own private signal, and sells the respective quantity at the actual market price.

We show that choices on information acquisition are strategic complements and that there are two types of equilibria: one where only the retailer decides to get informed and one where both the supplier and the retailer choose to increase the precision of their signals. The first type of equilibrium is due to the fact that contracts, which credibly signal the supplier's private information to be a high price signal, are costly in the sense that the supplier has to distort the quantities he offers. Comparing the implied signalling costs with the potential gains from lowering the retailer's information rent, the supplier decides to remain uninformed as long as the retailer's signal precision and thus his information rent is sufficiently small. By contrast and related to the second type of equilibrium, the supplier will choose to be informed, if the retailer's information rent is sufficiently high, 
i.e., if the retailer's signal precision is sufficiently high.

There is a vast literature on principal-agent models with endogenous information acquisition. Kessler (1998) analyzes an agent's incentive to get informed before the contracting stage. Similar to our model, this paper shows that it is not optimal for the agent to become perfectly informed as this would reduce the expected information rent. Considering the principal Bedard (2013), Kaya (2010), Nosal (2006) show that acquiring information may not be valuable for the principal as it leads to signalling costs in the contracting stage. Crémer et al. (1998), Crémer and Khalil (1994) examine the incentives of a principal to induce an agent to acquire additional information. In contrast to Kessler (1998) and to our model they assume that information gathering takes place after the principal has designed the contracts he offers, but before the agent decides, which contract to sign. We follow Kessler (1998) and Kaya (2010) by assuming that decisions about information acquisition are made prior to the contracting stage and that these decisions are observable. However, we allow both players to gather information and focus on the strategic interdependencies of these decisions.

Our paper is also closely related to the literature on information gathering within a supply chain which can be classified in terms of who acquires the information and how the information is used within the supply chain.

Guo and Iyer (2010) analyze the case, in which an upstream manufacturer can gather information on consumers' perceived product fit. The information acquisition takes place after contracting with a retailer and is affected by different information sharing mechanisms. They observe that the manufacturer does not have an incentive to be fully informed even if it is free of charge.

In contrast to this, there are several papers who consider a downstream retailer/ buyer investing in forecasting of demand or other uncertain parameters. Shin and Tunca (2010) show that if there is competition between retailers, the incentives of these retailers to invest in information acquisition are such that overinvestment occurs. If the investment is secret the overinvestment can be resolved by market based contracts, while in case of observable investments an uniform-price auction is required to solve that issue. A little closer to our model is the structure in Fu and Zhu (2010), where a single buyer acquires costly demand 
information after contract negotiations, but before ordering quantities. In their model, the added information might lead the informed party to improve its profits at the supplier's expense. They suggest a sharing mechanism for the cost and the information in order for the added information to lead to a Pareto-improvement. Guo (2009) addresses the question of forced versus voluntary disclosure of information and the effects of the disclosure rules on the firms' profits. It is shown that while forced disclosure actually harms the informed retailer and benefits the uninformed supplier, voluntary disclosure might restore the retailer's incentives to get informed in the first place.

Treating a similar problem, Kurtulus et al. (2008) consider a structure that is more similar to ours. Both supplier and retailer of a supply chain can acquire costly information about uncertain demand. However, the purpose of their paper is to identify benefits from sharing this individual information. They show that information sharing might benefit all members of the supply chain. In contrast to that our paper focuses on the strategic incentives to acquire private information.

The paper is structured as follows: Section 2 is concerned with the model framework. In section 3, we characterize the equilibrium contracts offered by the supplier for any given combination of private signals and precision choices. The decisions on information acquisition are analyzed in section 4 . Finally, section 5 concludes.

\section{Model}

We consider a three stage game with one supplier, one retailer and uncertain market conditions. In the first stage both players $i \in\{S, R\}$ can simultaneously choose the level of precision of the private signals about the actual market condition. While the selected signal precisions are observable, the signals themselves are private information. In the second stage, the supplier designs a menu of contracts, which he offers to the retailer. Then, the retailer chooses one of the contracts and offers the respective quantity on the market. The profit of the supplier is the transfer paid by the retailer minus the cost of producing the quantity specified in the contract, which has been chosen by the retailer. The retailer's profit is this quantity times the market price for the good, minus the transfer paid to the supplier. 
The retailer faces a perfectly competitive market. To capture uncertainty about market conditions, we assume that the market price for the product is $p \in\left\{p_{L}, p_{H}\right\}$ with $p_{H}=1$, $p_{L}=\alpha$ and $1 / 2<\alpha<1 .{ }^{1}$ Without loss of generality, we further assume that the commonly known probabilities for the high and the low price are

$$
\operatorname{Pr}\{p=1\}=\operatorname{Pr}\{p=\alpha\}=\frac{1}{2} .^{2}
$$

At the end of the first stage both players get private signals $\sigma_{i} \in\{H, L\}$ about the actual market price. Each player $i$ may choose his signal precision $\nu_{i} \in[1 / 2, \bar{\nu}]$ with $1 / 2<\bar{\nu} \leq 1$, without any costs. ${ }^{3}$ The probabilities for getting a correct signal are given by

$$
\nu_{i}=\operatorname{Pr}\left\{\sigma_{i}=H \mid p=1\right\}=\operatorname{Pr}\left\{\sigma_{i}=L \mid p=\alpha\right\}
$$

Correspondingly, the probabilities for getting a wrong signal are given by

$$
1-\nu_{i}=\operatorname{Pr}\left\{\sigma_{i}=H \mid p=\alpha\right\}=\operatorname{Pr}\left\{\sigma_{i}=L \mid p=1\right\}
$$

Note that $\nu_{i}=1 / 2$ implies that firm $i$ is completely uninformed. As mentioned, we assume that $\nu_{S}$ and $\nu_{R}$ are observable. The retailer's conditional probability that — given signal $\sigma_{S}$ - signal $\sigma_{R}=k$ reflects the true state of the world $k \in\{H, L\}$ can be written as

$$
\mu_{\sigma_{R} \sigma_{S}}=\operatorname{Pr}\left(p=p_{k} \mid \sigma_{R}, \sigma_{S}\right)=\left\{\begin{array}{l}
\frac{\nu_{R} \nu_{S}}{\left(1-\nu_{R}\right)\left(1-\nu_{S}\right)+\nu_{R} \nu_{S}}, \text { if } \sigma_{R}=\sigma_{S} \\
\frac{\left(1-\nu_{S}\right) \nu_{R}}{\nu_{S}\left(1-\nu_{R}\right)+\left(1-\nu_{S}\right) \nu_{R}}, \text { if } \sigma_{R} \neq \sigma_{S}
\end{array}\right.
$$

Turning to the second stage, the supplier chooses the menu of contracts that he offers. Each contract entails a fixed payment $T$ and a quantity $x$, which is produced by the supplier at a cost of

$$
C(x)=\frac{1}{2} x^{2}
$$

\footnotetext{
${ }^{1}$ Note that our results also hold in case of a strictly concave industry profit and the uncertainty relating to a shift in the demand curve.

${ }^{2}$ Allowing for different prior probabilities would complicate the analysis without leading to qualitatively different results.

${ }^{3} \bar{\nu}<1$ corresponds to assuming a convex cost function for information acquisition with $\lim _{\nu \rightarrow 1} C(\nu)=$ $\infty$
} 
Since we assume that this decision takes place after the supplier received his private signal, the offered menu can be conditioned on the supplier's signal $\sigma_{S}$

$$
C_{\sigma_{S}}=\left(C_{H \sigma_{S}}, C_{L \sigma_{S}}\right)=\left(\left(T_{H \sigma_{S}}, x_{H \sigma_{S}}\right),\left(T_{L \sigma_{S}}, x_{L \sigma_{S}}\right)\right) \text { with } \sigma_{S} \in\{H, L\}
$$

If $\nu_{S}=1 / 2$, the supplier's signal is not informative. Then the contracts he offers do not depend on $\sigma_{S}$ and correspond to pooling contracts, which do not signal any private information. On the other hand, $\nu_{S}>1 / 2$ may lead to signalling contracts $C_{H} \neq C_{L}$.

Our solution concept is the Perfect Bayesian Nash Equilibrium. To decrease the potentially high number of equilibria we make the following additional assumptions: Off equilibrium the retailer assumes that the supplier's signal is $\sigma_{S}=L$. Following the intuitive criterion we further assume that menu offer $C_{H}$ induces the retailer to belief $\sigma_{S}=H$, only if the supplier would not be better off by offering $C_{H}$ than $C_{L}$, if his signal was $L$.

In the next section, we characterize the equilibrium contracts. We then turn to the supplier's and the retailer's decisions on signal precision in the first stage of the game.

\section{Equilibrium Contracts}

We start with the case in which the signal of the supplier is $\sigma_{S}=L$ and then turn to $\sigma_{S}=H$.

If $\sigma_{S}=L$, the supplier offers $C_{L}=\left(C_{H L}, C_{L L}\right)$. The expected profit of the retailer, if he chooses contract $\left(T_{k L}, x_{k L}\right)$ with $k \in\{H, L\}$, can be written as $^{4}$

$$
\begin{aligned}
& E \Pi_{L L}^{R}\left(C_{k L}\right)=\left(1-\mu_{L L}\right) x_{k L}+\mu_{L L} \alpha x_{k L}-T_{k L}, \text { if } \sigma_{R}=L \\
& E \Pi_{H L}^{R}\left(C_{k L}\right)=\mu_{H L} x_{k L}+\left(1-\mu_{H L}\right) \alpha x_{k L}-T_{k L}, \text { if } \sigma_{R}=H
\end{aligned}
$$

Since $E \Pi_{H L}^{R}\left(C_{H L}\right)$ and $E \Pi_{L L}^{R}\left(C_{L L}\right)$ satisfy the single crossing property, the binding constraints for the optimal incentive compatible contracts $C_{H L}$ and $C_{L L}$ are given by

$$
E \Pi_{H L}^{R}\left(C_{H L}\right)=E \Pi_{H L}^{R}\left(C_{L L}\right) \text { and } E \Pi_{L L}^{R}\left(C_{L L}\right)=0
$$

\footnotetext{
${ }^{4}$ To simplify notation, we omit the exogenous parameter $\alpha$, as well as endogenous variables, which are taken as given at the respective stage of the game.
} 
The expected profit of the supplier given his signal was $\sigma_{S}=L$ can be written as

$$
\begin{aligned}
E \Pi_{L}^{S}\left(C_{L}\right) & =\left(1-\nu_{S}\right)\left(\nu_{R}\left(T_{H L}-\frac{1}{2} x_{H L}^{2}\right)+\left(1-\nu_{R}\right)\left(T_{L L}-\frac{1}{2} x_{L L}^{2}\right)\right) \\
& +\nu_{S}\left(\left(1-\nu_{R}\right)\left(T_{H L}-\frac{1}{2} x_{H L}^{2}\right)+\nu_{R}\left(T_{L L}-\frac{1}{2} x_{L L}^{2}\right)\right)
\end{aligned}
$$

Maximizing (2) subject to (1) we get

Lemma 1 The optimal menu $C_{L}^{*}=\left(C_{H L}^{*}, C_{L L}^{*}\right)$ is characterized by

$$
\begin{aligned}
& x_{H L}^{*}=\alpha+\mu_{H L}(1-\alpha) \\
& x_{L L}^{*}=\alpha+\left[1-\mu_{L L}-\frac{\nu_{S}\left(\left(1-\nu_{S}\right) \nu_{R}-\left(1-\nu_{S}\right)\left(1-\nu_{R}\right)\right)}{\left(\nu_{S} \nu_{R}+\left(1-\nu_{R}\right)\left(1-\nu_{S}\right)\right)^{2}}\right](1-\alpha)
\end{aligned}
$$

and

$$
E \Pi_{H L}^{R}\left(C_{H L}\right)=\left(\mu_{H L}-\left(1-\mu_{L L}\right)\right)(1-\alpha) x_{L L}^{*}
$$

Proof. See appendix.

Note that quantities $x_{H L}^{*}$ and $x_{L L}^{*}$ entail the standard result, of an optimal quantity for the "high type", i.e., $\sigma_{R}=H$, and a downward distorted quantity for the "low type", i.e., $\sigma_{R}=L$. While a retailer with $\sigma_{R}=H$ is able to make profit $E \Pi_{H L}^{R}\left(C_{H L}\right) \geq 0$, a retailer with $\sigma_{R}=L$ is left with 0 profit as it is depicted in (1).

With $\sigma_{S}=H$, the supplier offers $C_{H}=\left(C_{H H}, C_{L H}\right)$ and the retailer's expected profits are given by

$$
\begin{aligned}
& E \Pi_{L H}^{R}\left(C_{k H}\right)=\left(1-\mu_{L H}\right) x_{k H}+\mu_{L H} \alpha x_{k H}-T_{k L}, \text { if } \sigma_{R}=L \\
& E \Pi_{H H}^{R}\left(C_{k H}\right)=\mu_{H H} x_{k H}+\left(1-\mu_{H H}\right) \alpha x_{k H}-T_{k L}, \text { if } \sigma_{R}=H
\end{aligned}
$$

Again, the single crossing property holds and the binding constraints are given by

$$
E \Pi_{H H}^{R}\left(C_{H H}\right)=E \Pi_{H H}^{R}\left(C_{L H}\right) \text { and } E \Pi_{L H}^{R}\left(C_{L H}\right)=0
$$


The expected profit of a supplier with $\sigma_{S}=H$ can be written as

$$
\begin{aligned}
E \Pi_{H}^{S}\left(C_{H}\right) & =\nu_{S}\left(\nu_{R}\left(T_{H H}-\frac{1}{2} x_{H H}^{2}\right)+\left(1-\nu_{R}\right)\left(T_{L H}-\frac{1}{2} x_{L H}^{2}\right)\right) \\
& +\left(1-\nu_{S}\right)\left(\left(1-\nu_{R}\right)\left(T_{H H}-\frac{1}{2} x_{H H}^{2}\right)+\nu_{R}\left(T_{L H}-\frac{1}{2} x_{L H}^{2}\right)\right)
\end{aligned}
$$

In contrast to the case with $\sigma_{S}=L$, offering $C_{H}$ has to be credible, i.e., observing $C_{H}$ the retailer must be convinced that the supplier's signal was $\sigma_{S}=H$. Therefore, the menu $C_{H}$ has to satisfy the following credibility constraint

$$
\Delta\left(C_{H}, C_{L}^{*}\right):=E \Pi_{L}^{S}\left(C_{L}^{*}\right)-E \Pi_{L}^{S}\left(C_{H}\right) \geq 0
$$

Comparing (3) and (5) shows that there exists a unique $\widetilde{\nu}_{R}\left(\nu_{S}, \alpha\right) \in(0,1]$ such that $(5)$ is binding only for $\nu_{R} \leq \widetilde{\nu}_{R}\left(\nu_{S}, \alpha\right)$. Thus, we have

Lemma 2 The optimal contracts $C_{H}^{*}=\left(C_{H H}^{*}, C_{L H}^{*}\right)$ are characterized by

$$
x_{H H}^{*}=\alpha+\mu_{H H}(1-\alpha) \text { and } x_{L H}^{*}= \begin{cases}\widehat{x}_{L H}^{*}, & \text { if } \quad \nu_{R} \geq \widetilde{\nu}_{R}\left(\nu_{S}, \alpha\right) \\ \widehat{x}_{L H}^{*}+\Delta_{L H}^{*}, & \text { if } \quad \nu_{R} \leq \widetilde{\nu}_{R}\left(\nu_{S}, \alpha\right)\end{cases}
$$

where $\widehat{x}_{L H}^{*}$ and $\Delta_{L H}^{*}$ are given by

$$
\begin{aligned}
& \widehat{x}_{L H}^{*}=\alpha+\left[1-\mu_{L H}-\frac{\nu_{S}\left(\left(1-\nu_{S}\right) \nu_{R}-\left(1-\nu_{S}\right)\left(1-\nu_{R}\right)\right)}{\left(\left(1-\nu_{R}\right) \nu_{S}+\nu_{R}\left(1-\nu_{S}\right)\right)^{2}}\right](1-\alpha) \\
& \Delta_{L H}^{*}=\min \left\{\Delta_{L H}\left|\Delta\left(C_{H}, C_{L}^{*}\right)\right|_{x_{H}=x_{H}^{*}, x_{L H}=\widehat{x}_{L H}^{*}+\Delta_{L H}^{*}}=0\right\}
\end{aligned}
$$

Proof. See appendix.

Note that $x_{H H}^{*}$ and $\widehat{x}_{L H}^{*}$ correspond to the quantities for regular screening contracts $C_{H H}$ and $C_{L H}$ respectively. While $x_{H H}^{*}$ is undistorted, there is some distortion in $\widehat{x}_{L H}^{*}$ for any $\nu_{s}<1$. In addition, $\Delta_{L H}^{*}<0$ specifies the smallest additional downward distortion required for (5) to hold, if $\nu_{R} \leq \widetilde{\nu}_{R}\left(\nu_{S}, \alpha\right)$.

Intuitively, to ensure credibility of having received $\sigma_{S}=H$, the supplier has to decrease the quantity $x_{L H}$ as this lowers his profits, if the retailer gets signal $\sigma_{R}=L$ and therefore chooses $C_{L H}$-an event which is more unlikely with $\sigma_{S}=H$ as compared to $\sigma_{S}=L$. 


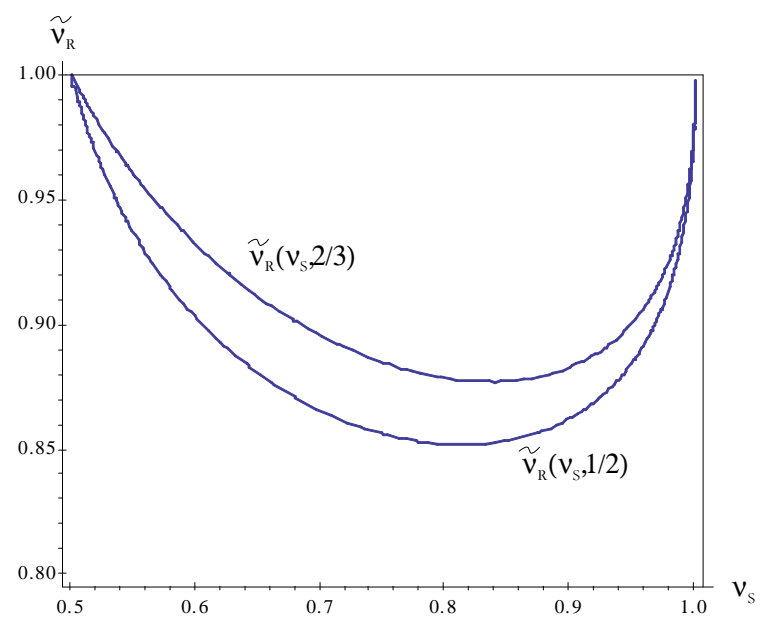

Figure 1: Critical values $\widetilde{\nu}_{R}\left(\nu_{S}, 1 / 2\right)$ and $\widetilde{\nu}_{R}\left(\nu_{S}, 2 / 3\right)$.

Thus, the increase in distortion of $x_{L H}^{*}$ allows the supplier to credibly signal $\sigma_{S}=H$, as the expected profit of $C_{H}^{*}$ would be reduced significantly, if $\sigma_{S}=L$. Figure 1 shows $\widetilde{\nu}_{R}\left(\nu_{S}, \alpha\right)$ for $\alpha=1 / 2$ and $\alpha=2 / 3$. Note that $\lim _{\nu_{S} \backslash 0.5} \widetilde{\nu}_{R}\left(\nu_{S}, \alpha\right)=1$. Thus, for $\nu_{S}$ being either rather high or low, additional distortion is required even for relatively high values of $\nu_{R}$.

Note further that we have $C_{H}^{*} \neq C_{L}^{*}$, if $\nu_{S} \neq 1 / 2$ and $\lim _{\nu_{S} \rightarrow 1 / 2}\left(C_{H}^{*}-C_{L}^{*}\right)=0$. Thus, $\nu_{S}=1 / 2$ leads to pooling contracts, which do not require the additional distortion to solve the supplier's credibility problem. $\nu_{S}=1$ on the other hand resolves all uncertainty for the supplier, but still requires additional distortion to credibly signal $\sigma_{S}=H$ to a retailer, who is less then perfectly informed.

\section{Choice of Signal Precision}

Turning to the first stage of the game, the expected profits of the supplier $E \Pi^{S}$ and the retailer $E \Pi^{R}$ can be written as

$$
\begin{aligned}
E \Pi^{S}\left(\nu_{S}, \nu_{R}\right)= & \frac{1}{2} E \Pi_{H}^{S}\left(C_{H}^{*}\right)+\frac{1}{2} E \Pi_{L}^{S}\left(C_{L}^{*}\right) \\
E \Pi^{R}\left(\nu_{R}, \nu_{S}\right)= & \frac{1}{2}\left(\left(1-\nu_{R}\right)\left(1-\nu_{S}\right)+\nu_{R} \nu_{S}\right) E \Pi_{H H}^{R}\left(C_{H H}^{*}\right) \\
& +\frac{1}{2}\left(\left(1-\nu_{S}\right) \nu_{R}+\nu_{S}\left(1-\nu_{R}\right)\right) E \Pi_{H L}^{R}\left(C_{H L}^{*}\right)
\end{aligned}
$$


Regarding the shape of these profit functions, we get the following result for the retailer

Lemma 3 A solution $\nu_{R}^{*}\left(\alpha, \nu_{S}\right)$ such that

$$
\frac{\partial E \Pi^{R}\left(\nu_{R}^{*}\left(\alpha, \nu_{S}\right), \nu_{S}\right)}{\partial \nu_{R}}=0 \text { and } \nu_{R}^{*}\left(\alpha, \nu_{S}\right)<\widetilde{\nu}_{R}\left(\nu_{S}, \alpha\right)
$$

only exists, if $\nu_{S}$ is sufficiently small. Furthermore, the retailer's marginal incentives for information acquisition have a downward kink at $\nu_{R}=\widetilde{\nu}_{R}\left(\nu_{S}, \alpha\right)$, i.e.,

$$
\lim _{\nu_{R} / \widetilde{\nu}_{R}\left(\nu_{S}, \alpha\right)} \frac{\partial E \Pi^{R}\left(\nu_{R}, \nu_{S}\right)}{\partial \nu_{R}}>\lim _{\nu_{R} \backslash \widetilde{\nu}_{R}\left(\nu_{S}, \alpha\right)} \frac{\partial E \Pi^{R}\left(\nu_{R}, \nu_{S}\right)}{\partial \nu_{R}}
$$

Finally, we have $\partial E \Pi^{R}\left(\nu_{R}, \nu_{S}\right) / \partial \nu_{R}<0$, if $\alpha, \nu_{R}, \nu_{S}$ are such that $x_{L H}^{*}=\widehat{x}_{L H}^{*}=\varepsilon$ with $\varepsilon>0$ but small.

Proof. See appendix.

The first part of the lemma implies that for $\nu_{S}=1 / 2$, the retailer's best response is not to be as informed as possible. This is simply due to the fact that the downward distortions of the quantities $x_{L L}^{*}$ and $x_{L H}^{*}$ are increasing in $\nu_{R}$ which impacts the retailer's expected profit negatively. The second part of lemma 3 indicates that $\widetilde{\nu}_{R}\left(\nu_{S}, \alpha\right)$ may be part of an equilibrium, if the kink of $E \Pi^{R}\left(\nu_{R}, \nu_{S}\right)$ is such that its left side is upward, while the right side is downward sloping. The final part guarantees that the retailer's best response never leads to $\widehat{x}_{L H}^{*}=0$, as the retailer would rather reduce the precision of his signal in order to prevent such an extreme distortion.

Turning to the supplier, we get

Lemma 4 The supplier's incentives for information acquisition are characterized by

$$
\begin{aligned}
& \left.\frac{\partial E \Pi^{S}\left(\nu_{S}, \nu_{R}\right)}{\partial \nu_{S}}\right|_{\nu_{S}=1 / 2}<0 \text { for } \nu_{R}<1 \text { and } \\
& \frac{\partial E \Pi^{S}\left(\nu_{S}, \nu_{R}\right)}{\partial \nu_{S}}>0 \text { for } \nu_{S} \text { and } \nu_{R} \text { sufficiently high. }
\end{aligned}
$$

Furthermore, $E \Pi^{S}\left(\nu_{S}, \nu_{R}\right)$ is strictly convex in $\nu_{S}$ for all $\nu_{R}, \nu_{S}$ such that $x_{L H}^{*}>0$.

Proof. See appendix. 
Lemma 4 guarantees by convexity of $E \Pi^{S}\left(\nu_{S}, \nu_{R}\right)$ that the supplier either chooses to get not informed at all or as much as possible. Intuitively, starting with $\nu_{S}=1 / 2$ an increase in $\nu_{S}$ forces the supplier to reduce $x_{L H}^{*}$ in order to satisfy the credibility constraint, which offsets any potential gains from being better informed. Although, if $\nu_{S}$ and $\nu_{R}$ are sufficiently high, the credibility constraint is not binding and the supplier can fully benefit from better information. Thus, if $\nu_{R}>\widetilde{\nu}_{R}\left(\nu_{S}, \alpha\right)$, the supplier has an incentive to be as informed as possible.

Combining lemma 3 and 4 and allowing for perfect information, we get that

$$
\frac{\partial E \Pi^{S}\left(\frac{1}{2}, \nu_{R}\right)}{\partial \nu_{R}}<0 \text { and } \frac{\partial E \Pi^{S}\left(1, \nu_{R}\right)}{\partial \nu_{R}}>0
$$

which indicates the complementarity of the precision choices. Solving for their equilibrium values, we can formulate the following proposition:

Proposition 1 Suppose $\bar{\nu}=1$. Then, there exist multiple equilibria with

$$
\nu_{S}^{*}=1 \text { and } \nu_{R}^{*} \in\left\{\nu_{R} \mid \alpha^{2}+4 \nu_{R}^{2}(3-\alpha(5-2 \alpha))-\left(2 \nu_{R}+10 v_{R}^{3}\right)(1-\alpha)^{2} \geq 1\right\}
$$

Proof. See appendix.

If $\bar{\nu}=1$, i.e., if the players can choose to be perfectly informed, the supplier is able to extract the entire rent from the retailer by choosing $\nu_{S}^{*}=1$ and the appropriate contracts. Thus, $E \Pi^{R}\left(\nu_{R}, 1\right)=0$, while for the supplier it holds that

$$
E \Pi^{S}\left(1, \nu_{R}\right)=\frac{2 \nu_{R}-1+\alpha^{2}}{4 \nu_{R}}>0
$$

If we restrict $\bar{\nu}<1$, the analysis of the equilibrium choices $\nu_{S}^{*}$ and $\nu_{R}^{*}$ is more involved since we have to distinguish whether $\nu_{R}$ is lower or higher then $\widetilde{\nu}_{R}\left(\nu_{S}, \alpha\right)$. Furthermore, we now get that two types of equilibria may coexist.

Proposition 2 Assume $\bar{\nu}<1$. Then, there exists a $\nu_{1}(\alpha) \in(1 / 2,1)$ such that the following decisions constitute an equilibrium

$$
\nu_{S}^{*}=\frac{1}{2} \text { and } \nu_{R}^{*}=\min \left\{\frac{5}{12}+\frac{1}{6(1-\alpha)}, \bar{\nu}\right\} \text {, }
$$




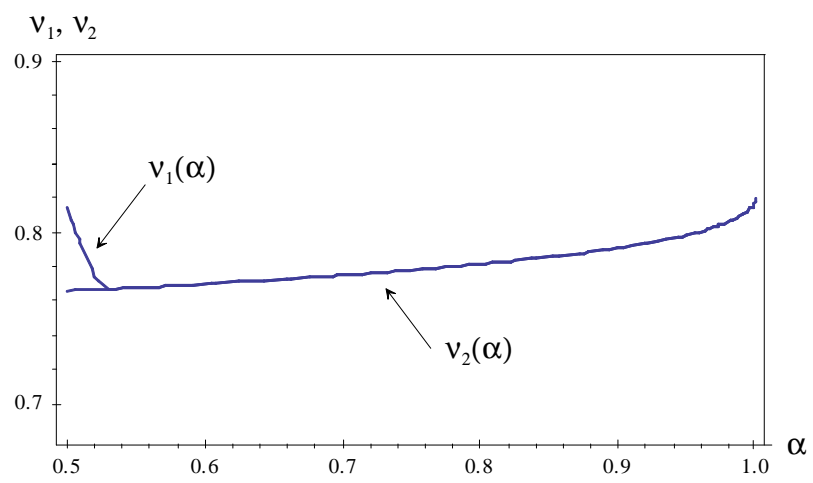

Figure 2: Equilibrium with $\nu_{S}^{*}=1 / 2\left(\nu_{S}^{*}=\bar{\nu}\right)$ below $\nu_{1}(\alpha)\left(\right.$ above $\left.\nu_{2}(\alpha)\right)$.

if $\bar{\nu} \leq \nu_{1}(\alpha)$.

Furthermore, there exists a $\nu_{2}(\alpha) \in(1 / 2,1)$ such that the following decisions constitute an equilibrium

$$
\nu_{S}^{* *}=\bar{\nu} \text { and } \nu_{R}^{* *}=\min \left\{\bar{\nu}, \max \left\{\widetilde{\nu}_{R}(\bar{\nu}, \alpha), \widehat{\nu}_{R}\right\}\right\}
$$

where $\widehat{\nu}_{R}$ is implicitly given by

$$
\frac{\partial E \Pi^{R}\left(\nu_{R}, \bar{\nu}\right)}{\partial \nu_{R}}=0 \text { for } x_{L H}^{*}=\widehat{x}_{L H}^{*}
$$

if $\bar{\nu} \geq \nu_{2}(\alpha)$

Proof. See appendix.

The graphs of $\nu_{1}(\alpha)$ and $\nu_{2}(\alpha)$ are shown in Figure 2. Note that we have $\nu_{1}(\alpha)>\nu_{2}(\alpha)$ for $\alpha$ sufficiently small, while $E \Pi^{S}\left(1 / 2, \nu_{R}^{* *}\right)=E \Pi^{S}\left(\bar{\nu}, \nu_{R}^{* *}\right)$ and $\nu_{R}^{* *}=\bar{\nu}$ lead to $\nu_{1}(\alpha)=\nu_{2}(\alpha)$ for $\alpha$ sufficiently high. Note also that for sufficiently small $\alpha$, both types of equilibria exist. To illustrate the different types of equilibria, assume that $\alpha=2 / 3$. Then, starting with $\bar{\nu}=1 / 2+\varepsilon$ as $\bar{\nu} \leq \nu_{1}(2 / 3)$, we have an equilibrium, with $\nu_{S}^{*}=1 / 2$ and $\nu_{R}^{*}=\bar{\nu}$ until $\bar{\nu}=$ $\nu_{1}(2 / 3)=\nu_{2}(2 / 3)$. If $\nu_{2}(2 / 3) \leq \bar{\nu}<\widetilde{\nu}_{R}(\bar{\nu}, 2 / 3)$, we have the equilibrium with both players acquiring as much information as possible, i.e., $\nu_{S}^{* *}=\bar{\nu}$ and $\nu_{R}^{* *}=\bar{\nu}$. For $\widetilde{\nu}_{R}(\bar{\nu}, 2 / 3) \leq \bar{\nu}$ $<1$, we get that $\nu_{S}^{* *}=\bar{\nu}$ and $\nu_{R}^{* *}=\widetilde{\nu}_{R}(\bar{\nu}, 2 / 3)$. Finally, if $\bar{\nu}=1$, we get the equilibria specified in proposition 1 . The corresponding equilibrium values $\nu_{R}^{*}, \nu_{S}^{*}$ as well as $\nu_{R}^{* *}, \nu_{S}^{* *}$ are shown in Figure 3. 


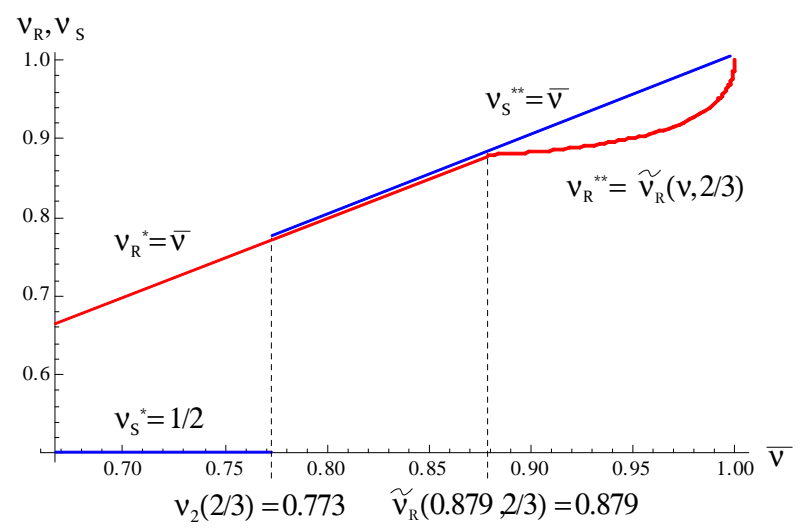

Figure 3: Equilibrium decisions for $\alpha=2 / 3$ and $\bar{\nu} \in[2 / 3,1)$.

The two types of equilibria show two different strategies for the supplier to cope with the credibility problem discussed above. When the retailer is not able to get well informed $(\bar{\nu}$ low), the supplier can refuse to get informed at all, as the distortion from the retailers information is low and the credibility problem can thus be avoided by offering pooling contracts. When the retailer can get well informed ( $\bar{\nu}$ high), distortion of $x_{L H}^{*}$ is required in order to be able to offer credible separating contracts. Thereby, the supplier is able to extract some information rent. As the information rent is relatively high, if the retailer is well informed, it is beneficial for the supplier to offer separating contracts, despite the required additional distortion of $x_{L H}^{*}$.

\section{Conclusion}

We have analyzed the incentives of a supplier and a retailer to acquire more accurate information about actual market conditions. Using a simple model we show that choices on information acquisition are strategic complements and that the supplier chooses to get informed, only if the information of the retailer is sufficiently precise even though the cost of information acquisition is 0 . If the maximum signal precision is sufficiently high, the retailer's information rent from acquiring additional information is relatively high as well. Thus, the supplier has stronger incentives to get informed as well and appropriate some of the information rent. If the maximum signal precision is sufficiently small, the retailer's information rent is rather small too. Thus, it becomes more attractive for the supplier to 
solve the credibility problem, which he faces, if he receives a signal for a high price, by not being informed and choosing pooling contracts.

\section{Appendix}

Proof of Lemma 1 Solving $E \Pi_{H L}^{R}\left(C_{H L}\right)=E \Pi_{H L}^{R}\left(C_{L L}\right)$ and $E \Pi_{L L}^{R}\left(C_{L L}\right)=0$ for $T_{H L}$ and $T_{L L}$, substituting in $E \Pi_{L}^{S}\left(C_{H L}, C_{L L}\right)$ and maximizing with respect to $x_{H L}$ and $x_{L L}$ leads to the stated results.

Proof of Lemma $2 \quad$ Solving $E \Pi_{H H}^{R}\left(C_{H H}\right)=E \Pi_{H H}^{R}\left(C_{L H}\right)$ and $E \Pi_{L H}^{R}\left(C_{L H}\right)=0$ for $T_{H H}$ and $T_{L H}$ and substituting in $E \Pi_{H}^{S}\left(C_{H H}, C_{L H}\right)$ we get

$$
\frac{\partial E \Pi_{H}^{S}}{\partial x_{H H}}=\frac{1-\nu_{R}-\left(1-2 \nu_{R}\right) \nu_{S}}{\nu_{R}+\left(1-2 \nu_{R}\right) \nu_{S}} \frac{\partial}{\partial x_{H H}} \Delta\left(C_{H}, C_{L}\right)
$$

Hence, the optimal quantity $x_{H H}^{*}$ is given by

$$
x_{H H}^{*}=\alpha+\mu_{H H}(1-\alpha)
$$

Turning to $x_{L H}$ assume first that with $x_{H H}=x_{H H}^{*}$ the constraint $\Delta\left(C_{H}, C_{L}^{*}\right) \geq 0$ is not binding. Then, $\partial E \Pi_{H}^{S} / \partial x_{L H}=0$ leads to $\widehat{x}_{L H}^{*}$.

Turning to the question whether $\Delta\left(C_{H}, C_{L}^{*}\right) \geq 0$ is binding, we use $C_{L}^{*}, x_{H H}^{*}$ and define $x_{L H}:=\widehat{x}_{L H}^{*}+\Delta_{L H}^{*}$. This allows us to write $\Delta\left(C_{H}, C_{L}^{*}\right)$ as

$$
\begin{aligned}
\left.\Delta\left(C_{H}, C_{L}^{*}\right)\right)\left.\right|_{x_{H H}=x_{H H}^{*}} & =\Phi\left(\nu_{S}, \nu_{R}, \alpha\right)-\frac{\left(1-2 \nu_{R}\right)^{2} \nu_{S}\left(1-\nu_{S}\left(3-2 \nu_{S}\right)\right)(1-\alpha)}{\left(\nu_{R}+\nu_{S}-2 \nu_{R} \nu_{S}\right)^{2}\left(1-\nu_{S}-\nu_{R}\left(1-2 \nu_{S}\right)\right)} \Delta_{L H} \\
& \left.-\frac{1}{2}\left[1-\nu_{S}-\nu_{R}\left(1-2 \nu_{S}\right)\right)\right] \Delta_{L H}^{2}
\end{aligned}
$$

where $\Phi\left(\nu_{S}, \nu_{R}, \alpha\right)$ is given by

$$
\Phi\left(\nu_{S}, \nu_{R}, \alpha\right)=\left.E \Pi_{H}^{S}\left(C_{H}\right)\right|_{x_{H}^{*}, x_{L H}=\widehat{x}_{L H}^{*}}-\frac{\left(2 \nu_{R}-1\right)^{3}\left(1-\nu_{S}\right)^{2} \nu_{S}^{2}\left(1-2 \nu_{S}\right)(1-\alpha)^{2}}{2\left(\nu_{R}+\nu_{S}-2 \nu_{R} \nu_{S}\right)^{4}\left(1-\nu_{S}-\nu_{R}\left(1-2 \nu_{S}\right)\right)^{2}} .
$$

Solving $\Phi\left(\nu_{S}, \nu_{R}, \alpha\right)=0$ for $\nu_{R}$ shows that there exists a unique solution $\widetilde{\nu}_{R}\left(\nu_{S}, \alpha\right) \in$ $[1 / 2,1]$ and that

$$
\Phi\left(\nu_{S}, \nu_{R}, \alpha\right) \lesseqgtr 0 \Longleftrightarrow \nu_{R} \lesseqgtr \widetilde{\nu}_{R}\left(\nu_{S}, \alpha\right)
$$


To determine the optimal quantity $x_{L H}$ for $\nu_{R}<\widetilde{\nu}_{R}\left(\nu_{S}, \alpha\right)$, note that

$$
\begin{aligned}
& \frac{\partial}{\partial x_{L H}}\left[E \Pi_{L}^{S}\left(C_{L}\right)-E \Pi_{L}^{S}\left(C_{H}\right)\right]_{x_{L H}=\widehat{x}_{L H}^{*}}<0 \text { and } \\
& \frac{\partial^{2}}{\partial x_{L H}^{2}}\left[E \Pi_{L}^{S}\left(C_{L}\right)-E \Pi_{L}^{S}\left(C_{H}\right)\right]=\left(1-2 \nu_{R}\right)\left(1-2 \nu_{S}\right)>0
\end{aligned}
$$

Furthermore, note that

$$
\frac{\partial^{2} E \Pi_{L}^{S}\left(C_{H}\right)}{\partial x_{L H}^{2}}=-\nu_{S}-\nu_{R}\left(1-2 \nu_{S}\right)<0
$$

Therefore we have that $E \Pi_{L}^{S}\left(C_{H}\right)$ as well as $\Delta\left(C_{H}, C_{L}^{*}\right)$ are symmetric around their maximum and minimum respectively. While there are two values for $\Delta_{L H}$, which satisfy $\Delta\left(C_{H}, C_{L}^{*}\right)=0$, we choose the one, which puts less distortion on $x_{L H}^{*}$. As it can be shown that

$$
\arg \min _{x_{L H}} \Delta\left(C_{H}, C_{L}^{*}\right)>\arg \max _{x_{L H}} E \Pi_{L}^{S}\left(C_{H}\right),
$$

we know by symmetry of the functions that the optimal distortion $\Delta_{L H}^{*}$ is given by

$$
\Delta_{L H}^{*}=\min \left\{\Delta_{L H}\left|\Delta\left(C_{H}, C_{L}^{*}\right)\right|_{x_{H H}=x_{H H}^{*}, x_{L H}=\widehat{x}_{L H}^{*}+\Delta_{L H}^{*}}=0\right\}
$$

Hence, it holds that $x_{L H}^{*}<\widehat{x}_{L H}^{*}$, whenever $\nu_{R}<\widetilde{\nu}_{R}\left(\nu_{S}, \alpha\right)$.

Proof of Lemma 3 The first part of the lemma follows from solving $\partial E \Pi^{R}\left(\nu_{R}, \nu_{S}\right) / \partial \nu_{R}=0$ with $x_{L H}^{*}=\widehat{x}_{L H}^{*}+\Delta_{L H}^{*}$ for $\nu_{S}$ as a function $\nu_{S}^{c}\left(\nu_{R}, \alpha\right)$. Numerical calculations show that $\nu_{S}^{c}\left(\nu_{R}, \alpha\right)$ attains its maximum at $\nu_{R} \approx 0.83$ and $\alpha=1 / 2$ and that this maximum is given by $\nu_{S}^{c}(0.83,1 / 2) \approx 0.625$. Hence, for $\nu_{S}>0.625$ there exists no solution $\nu_{R}^{*}\left(\alpha, \nu_{S}\right)$ such that $\partial E \Pi^{R}\left(\nu_{R}^{*}\left(\alpha, \nu_{S}\right), \nu_{S}\right) / \partial \nu_{R}=0$ and $\nu_{R}^{*}\left(\alpha, \nu_{S}\right) \leq \widetilde{\nu}_{R}\left(\nu_{S}, \alpha\right)$. The second results follow from evaluating $\partial E \Pi^{R}\left(\nu_{R}, \nu_{S}\right) / \partial \nu_{R}$ for $\nu_{R} \nearrow \widetilde{\nu}_{R}\left(\nu_{S}, \alpha\right)$ using $x_{L H}^{*}=\widehat{x}_{L H}^{*}+\Delta_{L H}^{*}$ and for $\nu_{R} \searrow \widetilde{\nu}_{R}\left(\nu_{S}, \alpha\right)$ using $x_{L H}^{*}=\widehat{x}_{L H}^{*}$. This leads to

$$
\begin{aligned}
\lim _{\nu_{R} \nearrow \widetilde{\nu}_{R}} \frac{\partial E \Pi^{R}\left(\nu_{R}, \nu_{S}\right)}{\partial \nu_{R}}>0 \text { and } \\
\lim _{\nu_{R} / \widetilde{\nu}_{R}\left(\nu_{S}, \alpha\right)} \frac{\partial E \Pi^{R}\left(\nu_{R}, \nu_{S}\right)}{\partial \nu_{R}}>\lim _{\nu_{R} \backslash \widetilde{\nu}_{R}\left(\nu_{S}, \alpha\right)} \frac{\partial E \Pi^{R}\left(\nu_{R}, \nu_{S}\right)}{\partial \nu_{R}} .
\end{aligned}
$$


The final part of the lemma can be proved by solving $\widehat{x}_{L H}^{*}=0$ for $\nu_{R}$ which leads to

$$
\nu_{R}^{c}=\frac{\nu_{S}\left(1+\nu_{S}-3\left(1+\nu_{S}\right) \alpha\right)-\sqrt{\left(1-\nu_{S}\right) \nu_{S}} \sqrt{(1-\alpha)\left(\nu_{S}\left(5-\nu_{S}(1-\alpha)+3 \alpha\right)-4 \alpha\right)}}{2\left(-1+2 \nu_{S}\right)\left(\nu_{S}-\left(1-\nu_{S}\right) \alpha\right)} .
$$

Substituting into $\partial E \Pi^{R}\left(\nu_{R}, \nu_{S}\right) / \partial \nu_{R}$ reveals

$$
\lim _{\nu_{R} \nearrow \nu_{R}^{c}} \frac{\partial E \Pi^{R}\left(\nu_{R}, \nu_{S}\right)}{\partial \nu_{R}}<0
$$

Proof of Lemma 4 The first two results follow from evaluating $\partial E \Pi^{S}\left(\nu_{R}, \nu_{S}\right) / \partial \nu_{S}$ and $x_{L H}^{*}=\widehat{x}_{L H}^{*}+\Delta_{L H}^{*}$ for $\nu_{S}=1 / 2$ and $x_{L H}^{*}=\widehat{x}_{L H}^{*}$ for $\nu_{R}>\widetilde{\nu}_{R}\left(\nu_{S}, \alpha\right)$. Numerical calculations show that $E \Pi^{S}\left(\nu_{R}, \nu_{S}\right)$ is strictly convex in $\nu_{S}$ as long as $x_{L H}^{*}>0$.

Proof of Proposition 1 Starting with the retailer, note that

$$
x_{L H}^{*}=\widehat{x}_{L H}^{*}+\Delta_{L H}^{*} \text { for } \nu_{S}=1 \text { and } E \Pi^{R}\left(\nu_{R}, 1\right)=0 .
$$

Considering the supplier, we get

$$
E \Pi^{S}\left(1, \nu_{R}\right)>E \Pi^{S}\left(\nu_{S}, \nu_{R}\right) \text { for all } \nu_{S} \in[1 / 2,1)
$$

as long as $\nu_{R}$ is such that

$$
\alpha^{2}+4 \nu_{R}^{2}(3-\alpha(5-2 \alpha))-\left(2 \nu_{R}+10 v_{R}^{3}\right)(1-\alpha)^{2} \geq 1
$$

According to lemma 1 and 2, the quantities in an equilibrium with $\nu_{S}^{*}=1$ are $x_{L L}^{*}=$ $x_{H L}^{*}=\alpha$ and $x_{H H}^{*}=1$ and are undistorted, while the credibility problem still leads to distortion in $x_{L H}^{*}$, which is

$$
\left.x_{L H}^{*}\right|_{\nu_{S}^{*}=1, \nu_{R}<1}=1-\frac{\sqrt{\nu_{R}-\alpha \nu_{R}^{2}}}{\nu_{R}} .
$$

Still, if $\nu_{R}^{*}=1$ as well, it also holds that $x_{H H}^{*}=x_{L H}^{*}$.

Proof of Proposition 2 Using lemma 4 we can prove the first part of the proposition by comparing the supplier's profits for $\nu_{S}=1 / 2$ and for $\nu_{S}=\bar{\nu}$ respectivelytaking into account the optimal decision of the retailer. Consider first $\nu_{S}=1 / 2$. Then, 
$\partial E \Pi^{R}\left(\nu_{R}, 1 / 2\right) / \partial \nu_{R}=0$ leads to

$$
\nu_{R}^{*}=\min \left\{\frac{5}{12}+\frac{1}{6(1-\alpha)}, \bar{\nu}\right\}
$$

as well as

$$
E \Pi^{S}\left(1 / 2, \nu_{R}^{*}\right)=\frac{1}{2}\left(2+5 \nu_{R}^{* 2}(1-\alpha)^{2}-(2-\alpha) \alpha-2 \nu_{R}^{*}(3-\alpha(5+2 \alpha))\right)
$$

Solving $E \Pi^{S}\left(1 / 2, \nu_{R}^{*}\right)=E \Pi^{S}\left(\bar{\nu}, \nu_{R}^{*}\right)$ for $\bar{\nu}$ leads to $\nu_{1}(\alpha)$ and the graph shown in Figure 2.

Turning to second part of the proposition, the retailer's best response $\nu_{R}^{* *}=$ $\min \left\{\bar{\nu}, \max \left\{\widetilde{\nu}_{R}(\bar{\nu}, \alpha), \widehat{\nu}_{R}\right\}\right\}$ is determined by whether $\widetilde{\nu}_{R}(\bar{\nu}, \alpha)$ or $\widehat{\nu}_{R}$ are feasible and by

$$
\lim _{\nu_{R} \backslash \widetilde{\nu}_{R}(\bar{\nu}, \alpha)} \frac{\partial E \Pi^{R}\left(\nu_{R}, \bar{\nu}\right)}{\partial \nu_{R}}\left\{\begin{array}{l}
<0 \Rightarrow \nu_{R}^{* *}=\widetilde{\nu}_{R}(\bar{\nu}, \alpha) \\
>0 \Rightarrow \nu_{R}^{* *}=\widehat{\nu}_{R}
\end{array}\right.
$$

Numerical calculations show that $E \Pi^{R}\left(\nu_{R}, \nu_{S}\right)$ is strictly concave in $\nu_{R}$ as long as $\nu_{R} \geq$ $\widetilde{\nu}_{R}(\bar{\nu}, \alpha)$ and that $\partial E \Pi^{R}\left(\nu_{R}, \bar{\nu}\right) / \partial \nu_{R}$ is strictly positive for all $\nu_{R} \leq \widetilde{\nu}_{R}(\bar{\nu}, \alpha)$ and $\nu_{S} \geq$ $\nu_{2}(\alpha)$ (see also the proof of lemma 3 ). Hence, the best response of the retailer is given by $\nu_{R}^{* *}$.

Considering the supplier, lemma 4 implies that the supplier's best response is either $\nu_{S}^{*}=$ $1 / 2$ or $\nu_{S}^{*}=\bar{\nu}$. Solving

$$
E \Pi^{S}\left(\frac{1}{2}, \nu_{R}^{* *}\right)=E \Pi^{S}\left(\bar{\nu}, \nu_{R}^{* *}\right)
$$

for $\bar{\nu}$ leads to $\nu_{2}(\alpha)$ shown in Figure 2 . 


\section{References}

Bedard, N. C. (2013). The strategically ignorant principal. ESEI Center for Market Design, Working Paper Nr. 18.

Crémer, J. and F. Khalil (1994). Gathering information before the contract is offered: the case with two states of nature. European Economic Review 38(3), 675-682.

Crémer, J., F. Khalil, and J.-C. Rochet (1998). Contracts and productive information gathering. Games and Economic Behavior 25(2), 174-193.

Fu, Q. and K. Zhu (2010). Endogenous information acquisition in supply chain management. European Journal of Operational Research 201 (2), 454-462.

Guo, L. (2009). The benefits of downstream information acquisition. Marketing Science 28(3), 457-471.

Guo, L. and G. Iyer (2010). Information acquisition and sharing in a vertical relationship. Marketing Science 29(3), 483-506.

Kaya, A. (2010). When does it pay to get informed? International Economic Review $51(2)$, $533-551$.

Kessler, A. S. (1998). The value of ignorance. The RAND Journal of Economics 29(2), $339-354$.

Kurtulus, M., M. Shor, and B. Toktay (2008). Investing in forecast collaboration. Working Paper, Vanderbilt University and Georgia Institute of Technology.

Nosal, E. (2006). Information gathering by a principal. International Economic Review $47(4), 1093-1111$.

Shin, H. and T. I. Tunca (2010). Do firms invest in forecasting efficiently? the effect of competition on demand forecast investments and supply chain coordination. Operations research 58(6), 1592-1610. 